\title{
Conciliación, elemento de pacificación en el desarrollo de la cultura de paz en Colombia
}

\author{
Conciliation, element of pacification in the development of the \\ culture of peace in Colombia
}

Gladis Ruiz Gómez*

Mireya Pérez Núñez**
*Universidad Simón Bolívar, Colombia

**Universidad de la Costa, Colombia

\section{Resumen}

Este artículo trata sobre la promoción de la cultura de paz, ya que es importante su difusión y aplicación para poner término a la violencia fratricida en Colombia. El objetivo de la investigación fue analizar la importancia de la implementación de uno de los Mecanismos Alternos de Solución de Conflicto (MASC) entre ellos la conciliación, como un elemento de pacificación que contribuye en el desarrollo de la política social de la cultura de paz en Colombia. El estudio se fundamentó en las siguientes fuentes: Constitución Política de Colombia (1991), Gorjón (2016), Molina (2014), Muñoz y López (2000), Martínez, (2000) y Galtung, (1998) entre otros. La investigación es documental enmarcada en el paradigma socio-crítico; el método empleado fue la hermenéutica. Se concluyó que los mecanismos alternos para la resolución de conflictos han dado buenos resultados en muchos países, por lo que se le debe dar una mayor promoción en Colombia para desarrollar el pluralismo judicial y no concebirlo como un inconveniente para la justicia tradicional, la cual se presenta como ineficiente para resolver controversias de tipo comunal.

Palabras clave: Conciliación, Cultura De Paz, Resolución De Conflicto, Pluralismo, Irenología.

\section{Abstract}

This article deals with the promotion of the culture of peace, since its dissemination and application

\section{Cómo citar}

Ruiz-Gómez, G., \& Pérez Nuñez, M. Conciliación, elemento de pacificación en el desarrollo de la cultura de paz en Colombia. MSC Métodos De Solución De Conflictos. Recuperado a partir de https://revistamsc.uanl.mx/index. $\mathrm{php} / \mathrm{m} /$ article/view/4 
is important to put an end to fratricidal violence in Colombia. The objective of the research was to analyze the importance of the implementation of one of the Alternative Conflict Resolution Mechanisms (MASC), including conciliation, as an element of pacification that contributes to the development of the social policy of the culture of peace in Colombia. The study was based on the following sources: Political Constitution of Colombia (1991), Gorjón (2016), Molina (2014), Muñoz and López (2000), Martínez, (2000) and Galtung, (1998) among others. The research is documentary framed in the socio-critical paradigm; the method used was hermeneutics. It was concluded that alternative mechanisms for conflict resolution have given good results in many countries, so it should be given greater promotion in Colombia to develop judicial pluralism and not conceive it as an inconvenience to traditional justice, which is presented as inefficient to resolve communal disputes.

Key Words: Conciliation, Culture Of Peace, Resolution Of Conflict, Pluralism, Irenology.

\section{INTRODUCCIÓN}

El tema de la paz ha sido analizado a lo largo de los siglos por los más grandes filósofos, desde Platón, en adelante; en su sentido tradicional la paz se interpreta como la situación y relación mutua de quienes no están en guerra, igualmente se trata de la tranquilidad. En los Estados, en contraposición a la beligerancia se acuerda entre las partes en conflictivos tratados de paz para poner fin a la guerra. La idea de establecer la paz tiene orígenes inmemoriales y en diferentes creencias, por ejemplo así lo estipulaban los registros religiosos del judaísmo (Genesis 9: 6), cristianismo (Mateo 5: 9), entre otros.

El presente artículo se plantea la problemática sobre la importancia de la implementación de uno de los Mecanismos Alternos de Solución de Conflicto (MASC). Así, ¿Podría la conciliación convertirse en un elemento de pacificación y contribuir al desarrollo de una política social, para la cultura de paz en
Colombia? En este sentido, se justifica por la importancia de la construcción de una cultura de paz que sea capaz de permitir una renovación del pensamiento requerido para el postconflicto, motivo por el cual urge la necesidad de fortalecer las políticas sociales para tal efecto, que contrarresten estructuras socio-económicas de atraso, promuevan nuevas formas de pensamiento y destierren el ideario de la cultura de la confrontación.

Entre los autores que han estudiado la Conciliación, como un elemento clave de pacificación para el desarrollo de la cultura de paz, se encuentran los siguientes: CabeIlo-Tijerina, P. y Quiñones, K. (2019), CabeIlo, Carmona, Gorjón, Iglesias, Sáenz y Vázquez (2016); Audrerie, (2015); Molina, (2014); López, (2011); Fisas (2010); Martínez (2000); Muñoz y López, (2000); Galtung (1998), Jares (1999); Zapata (2012). Además, abordan la conciliación como mecanismo estratégico para la paz leyes y documentos del Ministerio de Justicia y del Derecho de Colombia. 
El objetivo del presente trabajo es analizar la importancia central de una efectiva implementación de la conciliación como parte del catálogo de los MASC y un elemento de pacificación que contribuye al proceso de desarrollo de la política social de la cultura de paz en Colombia. La investigación se estructura de la siguiente manera: 1. Conceptualización de la cultura de paz desde la Irenología; 2. Clases de paz; 3. Mecanismos Alternos de Solución de Conflicto (MASC); la conciliación y, por último, se presentan las reflexiones finales.

\section{MARCO TEÓRICO- ANÁLISIS}

\section{CONCEPTUALIZACIÓN DE LA CULTU- RA DE PAZ DESDE LA IRENOLOGÍA}

Al explicar el contexto histórico de la cultura de paz, nos remitimos a la etimología del sustantivo femenino de cultura procede del latín cultūra (Diccionario de la Real Academia Española [RAE], 2006, p. 902) y se ha tomado para la investigación el significado siguiente: "conjunto de modos de vida y costumbres, conocimientos y grado de desarrollo artístico, científico, industrial, en una época, grupo social, etc."

En cuanto a la paz, el sustantivo femenino paz proviene del latín pax, pacis, el cual tiene diversas acepciones, para este caso específico se acepta el significado que más corresponde con el objeto de estudio; "situación en la que no existe lucha armada en un país o entre países" (RAE, 2006). No obstante, en el caso colombiano, se analiza la violencia arraigada dentro de sus fronteras (conflicto interno).
Al respecto, el origen del concepto cultura de paz, como lo recoge originalmente la Organización de las Naciones Unidas para la Educación, la Ciencia y la Cultura (UNESCO), se remonta de acuerdo con Giesecke (1999) al Perú donde se trató de desarrollar una educación para la paz en la década de 1980 debido a la guerra interna desatada por el grupo guerrillero de Sendero Luminoso. No obstante, fue la Asociación Peruana de Estudios para la Paz creada en 1983 un organismo no gubernamental, que acuñó de manera categórica un concepto de cultura de paz, a través de su presidente, el profesor universitario y sacerdote Felipe MacGregor quien, planteó la definición de Cultura de paz Dávila (2014: 147):

La paz es dinámica. La paz es la solución justa y ni violenta de los conflictos. Genera un equilibrio en la interacción social, de tal manera que todos los miembros de la sociedad puedan vivir en relaciones armoniosas uno con el otro.

De acuerdo con esta definición que data de 1985, según Capella, y Tueros (2005) así como, Dávila (2014) era novedosa para la época, ya que no definía a la paz como la ausencia de guerra, sino que le agregó elementos como la justicia y la libertad. Posteriormente, Mac Gregor y otros expertos redactaron el Manifiesto de Sevilla sobre la Violencia en 1986, el cual fue tomado en cuenta por la Declaración de Yamusukro en la Mente de los Hombres de 1989 en Costa de Marfil la cual señala que la paz es: 
esencialmente el respeto de la vida; el bien más preciado de la humanidad; no es sólo el término de los conflictos armados; un comportamiento; una adhesión profunda del ser humano a los principios de libertad, justicia, igualdad y solidaridad entre todos los seres humanos; es una asociación armoniosa entre la humanidad y el medio ambiente.

A partir de numerosos aportes de muchos países sobre la paz, la UNESCO propuso por primera vez un concepto de la cultura de paz en el Congreso Internacional organizado por esta Organización de las Naciones Unidas (ONU) sobre la Paz en la Mente de los Hombres, celebrado en Yamusukro, este concepto de tendencia universalizante tiene 32 años de creado y éste ha cambiado la forma en que entendemos la paz.

La cultura de la paz es una visión donde, la paz no es el cese del conflicto armado, es una manera de comportarse de convivir, de esta manera deja de ser un concepto simple, que se asocia exclusivamente al hecho bélico, debería extenderse y ser contemplado como un modo de vida. De esta manera, la UNESCO considera la cultura de paz como una acción que a través de la educación por la paz permite acabar con la guerra, pero también una ciencia comprometida con el ser humano y el planeta y la cultura desde el punto de vista de la interculturalidad y el pluralismo cultural, aunado a este objetivo se promueve la colaboración entre las naciones, a fin de asegurar el respeto universal a la justicia, a la ley, a los derechos humanos y a las libertades fundamentales.
Cabe destacar que para definir la categoría cultura de paz: se debe tener en cuenta, que no existe una noción única y precisa de lo que ésta significa, igualmente sobre lo que significa la paz, Jares (1999), citado por Cabello, Carmona, Gorjón, Iglesias, Sáenz y Vázquez (2016), opina que existen distintas acepciones que se pueden encontrar en el ámbito académico y científico, así como, en su evolución histórica, por lo tanto, no se puede concluir en una sola definición, porque se convertiría en hegemónica con respecto a otras definiciones o concepciones de otras sociedades.

Ahora bien, la idea de establecer la paz se ha originado desde tiempos remotos y en diferentes creencias, sin embargo, desde el fin las dos guerras mundiales del siglo XX y del posible caos atómico, la paz y la guerra comenzaron a ser consideradas materia de la ciencia, según Molina (2014) Bouthoul creador en 1942 del neologismo polemología es decir, fue precursor en el estudio de la guerra como fenómeno social.

Por otra parte, el periodista belga Paul Levi creó en 1950 el término opuesto al neologismo del estudio de la guerra, es decir la irenología, que es el estudio de la paz, ésta se completa con el estudio de la guerra, de allí que para el desarrollo de ambas ciencias, se han utilizado de acuerdo a Muñoz y López (2000) las contribuciones de otras disciplinas desde un enfoque interdisciplinar y transdisciplinar abierto, con capacidad de integrar y ganar en capacidad de relación e integración de las diversas otras variables presentes. Por lo que, la concepción de la paz no se debe considerar sólo como la ausencia de guerra. 
En cuanto, a la irenología, ésta tomó impulso a partir de los movimientos pedagógicos surgidos entre las dos guerras mundiales que abordaron según Muñoz y López (2000) un tipo de educación y formación más democrática, junto a los movimientos no-violentos haciendo grandes esfuerzos en la capacitación para desarrollar los primeros pasos de la Investigación para la Paz. Si bien es cierto, en sus comienzos, la investigación para la paz "permitió desarrollar mucho más la polemología que la irenología, en gran medida debido que el fenómeno de la guerra y sus asociados debían ser explicados de manera racional, lógica y científicamente" (p. 21) para no sólo diagnosticarla sino evitar su proceso, de esta manera, de acuerdo con los autores antes citados, la irenología tomó una gran vitalidad y acabó por despegar como disciplina científica.

En cierta manera la irenología ha contribuido según Muñoz y López (2000) "al declive de la ideología de la neutralidad científica y ha creado una sensibilidad hacia las posibles utilizaciones y manipulaciones de signo represivo de sus investigaciones" (p. 21). Además, es una disciplina que cuenta con un campo bastante extenso al nutrirse de otras ciencias como: la Politología, la Sociología, la Historia, las Relaciones internacionales, el Derecho entre otras, de esta manera comprende un campo de acción de carácter interdisciplinario y transdisciplinario.

También cabe señalar, que, en la antigüedad al conquistar los romanos a los griegos, se dio un gran intercambio cultural desde todo punto de vista, igualmente, sucedió en cuanto a las concepciones que se tenían so- bre la paz. El sentido de estado de armonía que los antiguos griegos le dieron a la paz según Cabello, et al., (2016, p. 6):
"estableció los cimientos sobre los cua- les se erigiría la noción de paz que en la actualidad persiste en occidente: es decir, la paz era ese preciso momen- to en el que cesaban las hostilidades entre los ejércitos combatientes de la ciudades-Estado griegas".

En Roma se denominó a un período histórico relativamente largo como la paz romana, que, sin embargo, en investigaciones de Muñoz y López (2000) ocultaba situaciones de violencia (este período abarcó desde el primer emperador Augusto, hasta después de la muerte del emperador Marco Aurelio). La concepción romana de la paz era mantenerse dentro de la legalidad de sus leyes que muchas veces no eran equitativas, cuyo lema era "mantener la paz de la ley y el orden". (Cabello et al. 2016, p. 6).

Ahora bien, uno de los pensadores que más ha teorizado sobre la irenología en el siglo XX y XXI según Cabello et al. (2016, p.17) es Galtung, su tesis radica en: "que las culturas violentas y las estructuras violentas no se pueden solucionar mediante la violencia, pues ello llevaría a nuevas estructuras violentas y además reforzaría una cultura bélica".

Para Galtung (1981), paz cuando en la sociedad se cumplen la satisfacción de las necesidades: de bienestar (no a la violencia clásica), libertad, identidad, sobrevivencia (no hay privación de necesidades materia- 
les básicas), sin represión (respeto por los derechos humanos), limitación o condicionamiento de la personalidad, impuestos al individuo o a la colectividad por factores externos sociales, económicos o culturales, la lista no es exhaustiva y bien se podrían agregar muchas más.

Gracias a los aportes de la irenología, se ha llegado al entendimiento de que la paz no puede ser definida solamente como la ausencia de guerra, sino como un concepto positivo el cual incluye la justicia, el desarrollo económico y social equilibrado, y el conocimiento y respeto mutuo entre las naciones; (Terminski, 2010) así como, todos los demás factores que contribuyan a la desaparición de la violencia estructural, la pobreza, la desigualdad, el racismo, la xenofobia, los prejuicios y estereotipos culturales negativos, así como el militarismo.

Es interesante recalcar que actualmente la paz es considerada como un valor universal, es por lo que las naciones del orbe expresan profunda preocupación por la persistencia y la proliferación de la violencia y los conflictos en diversas partes del mundo, de allí la importancia de desarrollar programas que promuevan el respeto pleno y la promoción de todos los derechos humanos y las libertades fundamentales. (Adams, 1992).

\section{CLASES DE PAZ}

Es necesaria la edificación de la paz como un instrumento para la creación de una nueva ideología con nuevos valores de solidaridad, amor, conmiseración por el prójimo, entre otros, siendo éstos de acuerdo con
Muñoz y López (2000) "las vías de regulación pacífica de los conflictos que se conviertan en la opción principal ante todas las situaciones presentes y futuras" (p. 49). Igualmente, es necesario desarrollar y perfeccionar el análisis de las causas, estructuras y dinámicas de las situaciones de conflicto y las vías de regulación de los mismos; enunciar las instancias en las que se producen los laboratorios de violencia; investigar la relación causal y analizar los mecanismos de mediación para una resolución amigable, con acuerdos equitativos entre las partes en conflictos.

En los años sesenta, Galtung (1995) fue pionero en los estudios para la paz, es quien introduce los conceptos de paz negativa, igualmente, crea la categoría paz positiva. De esta manera, la paz es concebida en dos perspectivas con un lado positivo y otro negativo.

\subsection{La paz negativa}

Galtung (1968) es quien delinea el concepto de paz negativa que implica la ausencia de guerra. En cuanto a la estructura dada por Galtung (1998, p. 15) referente a los conceptos de violencia, que impiden la paz, (paz negativa) los categoriza en tres: directa, cultural y estructural. El primer tipo de violencia se refiere a la agresión siendo su máxima expresión la guerra. Mientras que la violencia cultural "es la suma de todos los mitos, de gloria y traumas y demás que sirven para justificar la violencia directa" (p. 16) de esta manera se legitima la violencia como forma de resolver los conflictos.

Por último, define la violencia estructural, como "la adicción de todos los choques in- 
crustados en las estructuras sociales, y mundiales, cimentadas solidificadas, de tal forma que los resultados injustos, desiguales, son casi inmutables" (Galtung, 1998, p. 16) esto impide que las personas sean tratadas con equidad, vulnerando con todo esto los derechos del ciudadano y diluyéndose las obligaciones del Estado y de la comunidad internacional.

\subsection{La paz positiva}

De igual manera, Galtung (1998) crea la categoría paz positiva relacionada con el respeto a la vida, la justicia social, los derechos humanos, es la ausencia de todo tipo de violencia, donde los tipos de violencia señalados por él deben superarse a través de la democracia, la política de la no violencia o convertir los círculos viciosos en círculos virtuosos. Complementando a Galtung, Fisas (2010), agrega que la paz es un proceso para consolidar una nueva manera de ver, entender y vivir en el mundo, empezando por el propio ser y continuando con los demás, horizontalmente, formando una red, dando confianza, seguridad, autoridad a las personas y a las sociedades, promoviendo el intercambio mutuo, superando desconfianzas y diferencias desde una perspectiva local y global.

\subsection{Diferentes definiciones de paz}

Por otra parte, a las nociones dicotómicas de las formas de paz de Galtung, se han agregado otros tipos de paz a través de la historia como: la paz silenciosa, paz innominada, paz imperfecta, la paz antropocéntrica que ha obviado la conflictividad biocéntrica, la paz neutra y la paz parcial.
En cuanto a la paz silenciosa dice Muñoz y López (2000) que es la alianza, los intercambios tanto físico como materiales e inmateriales entre pueblos que comerciaban dándose así una especie de socialización, de alianzas matrimoniales y a la vez estos intercambios favorecían la satisfacción de las necesidades de otras comunidades, "las buenas relaciones de vecindad, la hospitalidad, la cooperación, los acuerdos, los pactos, entre otros. La paz silenciosa forma largas cadenas y redes que facilitan el intercambio y el enriquecimiento cultural de los habitantes de las sociedades vecinas" (p. 30). Un ejemplo de esta paz silenciosa se puede notar en la frontera colombo venezolana, la frontera está cerrada a los intercambios de transporte, más no a las relaciones humanas y comerciales de los ciudadanos de ambos países.

En cuanto a la paz innominada, que no tiene nombre especial (RAE, 2006):

no medida ni en el tiempo ni en espacio, se difumina en la representación geométrica de los «grandes acontecimientos», entre los que ocupan un lugar relevante las guerras, las conflagraciones, entre otros, que aparecen jaloneando la línea del transcurrir histórico que, de esta manera, representan y distorsionan de manera aparentemente continuada a la perspectiva de los ritmos sociales (p.31).

La paz imperfecta, denominada de esta manera, por Muñoz y López (2000) porque nunca se da una paz absoluta, perfecta, es imperfecta porque "no está presente en todos 
los espacios sociales, sino que convive con el conflicto y las distintas alternativas que se dan socialmente a éste para regularlo" Muñoz y López (2000, pp. 46-47). A pesar de que se den propuestas de regulación pacífica, las propuestas y acciones violentas puede que también estén casi siempre presentes, estos tipos de pases están ampliamente señaladas en la historiografía tradicional. Al respecto, Harto de Vera, (2016) agrega que la paz imperfecta es un proceso inacabado, no obstante, este proceso de construcción de la paz es incesante y perdurable, siendo tan permanente como "(...) la presencia del conflicto en la vida humana" (p. 143).

Otro elemento muy importante agregado al tratamiento de la paz, por López (2011), es una crítica al estudio de ésta desde una óptica antropocéntrica obviando la conflictividad biocéntrica de manera que "el conflicto no sólo se entienda como las divergencias de criterios, intereses, emociones y sentimientos entre los seres humanos"( López, p. 91), el conflicto es más complejo ya que, envuelve otro tipo de conflictividad que involucra todas las formas de vida y el conjunto natural; de ahí que sea dable hablar de una cultura de paz como un cultivo de las relaciones con los otros y la naturaleza (Martínez, 2000).

La paz neutra de Jiménez (2014), se refiere a neutralizar la violencia cultural y/o simbólica, que procura accionar a través del diálogo, la búsqueda de consensos, así como el desarrollo de la criticidad en la educación integral, interdisciplinaria y neutral para la paz, con la finalidad de neutralizar los patrones de violencia de cada sociedad para organizar las interrelaciones sociales en su conjunto.
Por otra parte, otro tipo de paz observado precisamente en Colombia es una paz que se puede llamar convencional o como (Ruiz) la ha definido es una paz parcial, puesto que, se está en espera de nuevos acuerdos de paz con los grupos guerrilleros disidentes de las Fuerzas Armadas Revolucionarias de Colombia (FARC) y el Ejército de Liberación Nacional (ELN). Por lo que, no se puede afirmar que existe ausencia de conflicto armado en Colombia, se dice que son un pequeño grupo, pero tienen según Johnson (2017), el control de una zona extensa del país como el Guaviare, que hará difícil el sometimiento de los subversivos por lo impenetrable de dicha zona, sin infraestructura vial y abandonada a su suerte.

Los grupos guerrilleros disidentes no aceptan los acuerdos llevados a cabo por la dirigencia de las FARC y el gobierno colombiano, por lo que se puede visualizar que los logros para la paz en Colombia no son integrales, porque existe un conjunto de valores indeseables, actitudes poco amistosas, estilos de vida proclives al no respeto a la vida y en la práctica a favor de la violencia en ciertas regiones importantes del territorio colombiano, donde la libertad de expresión no existe, la pena de muerte es aplicada de manera sumaria in situ, sin derecho a la defensa y los principio democráticos no tienen vigencia. En cuanto a la tolerancia, la solidaridad, el pluralismo y el entendimiento son principios todavía de alcance limitado en la Colombia postguerrilla.

Por consiguiente, la sociedad colombiana está a la espera de nuevos acuerdos de paz con los grupos guerrilleros disidentes, a fin 
de obtener una paz completa y duradera en cuanto a conflictos armados se refiere, de allí la importancia de seguir invirtiendo en el desarrollo de la cultura de paz como política social aplicada en la resolución de los conflictos. Donde la conciliación, es uno de los más necesarios mecanismos alternos de solución de conflictos. Porque considera elementos importantes de pacificación para el desarrollo de la cultura de paz en este país sudamericano ya que, es de vital trascendencia en este esfuerzo permanente por fortalecer a la paz.

Además, Colombia contempla en la (Constitución Política, 1991) específicamente en el artículo 22 que "La paz es un derecho y un deber de obligatorio cumplimiento". De esta manera se constata que el Estado considera el mantenimiento de la paz como un hecho primordial. No obstante, la paz es un concepto que aborda distintos aspectos de la sociedad, por tanto, la disidencia considera que no podría haber paz, si existe en Colombia desigualdad, sufrimiento, mortalidad, alienación, represión, exclusión política, discriminación étnica entre otros, sería lo que llama Galtung (1998), la paz negativa.

\section{MECANISMOS ALTERNOS PARA LA SOLUCIÓN DE CONFLICTOS (MASC)}

Existen componentes estatales formales y no formales para la resolución de conflictos en las comunidades, a fin de contribuir al mejoramiento del acceso a la justicia de todos los ciudadanos, ya que a través de la promoción y el uso de los MASC se fomenta la cultura de paz. Los MASC, no son empleados comúnmente, puesto que la mayor parte de la ciudadanía aún los desconoce y se dirigen mecánicamente hacia la justicia tradicional. De la cual sabemos de su saturación, pobres resultados y sus costos son demasiados onerosos. Estos mecanismos son están reconocidos en la legislación del Estado colombiano, sin embargo, es un proceso apenas iniciado en los años noventa.

En 1991 al entrar en vigor la nueva Constitución Política de Colombia, se amplía el espectro para la resolución de conflictos, modificando el entorno legal e introduciendo nuevas instituciones jurídicas que tienen la intención de superar los problemas de acceso al sistema judicial y despresurizar la carga de trabajo de juzgados y tribunales. Surge así la posibilidad para que los particulares actúen como conciliadores o árbitros, habilitados por las partes para fallar en derecho o equidad, como alternativas para que la comunidad y los ciudadanos puedan resolver sus conflictos de manera expedita, pacífica y consensuada. En el mismo sentido se crea la figura de los Jueces de Paz, como jurisdicción rápida y gratuita, accesible para todos los ciudadanos que tramiten conflictos menores.

En efecto, la nueva constitución colombiana incorporó órganos que administran justicia, a los particulares y a las autoridades indígenas y comunidades afrodescendientes, restableciendo la capacidad de la sociedad para participar en el proceso de administración de justicia, con la aplicación de la norma el como un instrumento de fuerte contenido social. Todo esto permite legitimar a los MASC como la conciliación, que se convierte en en un aliado fundamental de la 
descongestión judicial, una herramienta privilegiada extraprocesal previa al litigio y un medio procesal de terminación anticipada a la judicialización del litigio.

El origen etimológico del concepto conciliación se encuentra en el latín conciliatio, -ōnis (RAE, 201) que en derecho significa un acuerdo de los litigantes para evitar un pleito o desistir del ya iniciado. Igualmente, conciliación encarna según el (Diccionario Larousse, 201). “Acciones destinadas a restablecer el buen entendimiento entre las personas cuyas opiniones o intereses se oponen: Buscar la conciliación entre las partes opuestas".

En cuanto a la mediación ésta proviene del latín mediatio, -ōnis., significado que en derecho significa la actividad desarrollada por una persona de confianza de quienes sostienen intereses contrapuestos, con el fin de evitar o finalizar un litigio. Para ubicar los antecedentes de la conciliación es necesario mencionar que la mediación, a diferencia de la conciliación, según Zapata (2015, p. 23) "no está ampliamente enmarcada por la ley colombiana. Además, la mediación es una creación de doctrina, mientras que la conciliación tiene un estatus constitucional".

La fuente de la mediación en Colombia se remonta según Audrerie (2015) a los jueces de paz en funciones desde 1834. Estos últimos, eran personas que, sin ser jueces de la república, sirvieron de mediadores para resolver todo tipo de conflictos entre ciudadanos. Su intervención fue un paso preliminar antes de acudir directamente a la justicia ordinaria.
Mientras que Lopera (2011), la crítica en su análisis, ya que, a pesar de la reglamentación desarrollada, ésta no se ha orientado hacia la realización de la justicia como un fin en sí mismo, más bien como solución a un problema de acceso y congestión judicial la cual desconoce los procesos de construcción participativa, dejando de lado las interculturalidades y escenarios donde los diferentes actores interactúan para lograr alcanzar una verdadera justicia.

Justamente, la sociedad civil ha manifestado su rechazo a la comisión de actos específicos de violación de derechos humanos en Colombia, según la International Crisis Group (2010) citada por Valdivieso (2012) tienen un nivel alto de gravedad, por lo que se requiere de una decidida participación del Estado en la ejecución de acciones políticas encaminadas a garantizar la protección de éstos en la era del postconflicto armado. Además, según Cabello-Tijerina, y Quiñones (2019), la actual paz en Colombia exige mantener vigente el acuerdo celebrado entre el gobierno y las FARC, de allí la importancia de potenciar el desarrollo los MASC entre la población colombiana.

\subsection{Las políticas públicas y la concilia- ción}

Tomando en consideración lo planteado por Barenboim (2012), sobre las políticas públicas que surgen como espacios centrales de mediación y resolución de conflictos, además, de ser una interconexión de procesos para la redefinición de soluciones y son consideradas igualmente como propuestas de regulación pública. 
Estas políticas están asociadas a los asuntos inherentes a los gobiernos y los sistemas políticos, a las actividades de las instituciones públicas dirigidas a influenciar la vida de los ciudadanos, y que tienen como elemento determinante su procesamiento desde la responsabilidad de las instituciones y organizaciones gubernamentales, como lo afirman Maggiolo y Perozo (2007).

En cuanto a las sociedades que alcanzan la etapa de posconflicto, según Ugarriza (2013) y Cabello-Tijerina, y Quiñones (2019), éstas deben trabajar para la creación de espacios para su desarrollo, y para lograr esos fines, deberán enfrentar la opción de construcción de la paz, lo cual implica lograr la estabilidad política. Para lograr la paz, se necesita la institucionalización de los mecanismos legales existentes como única vía para dirimir controversias sobre decisiones públicas e igualmente se debe incluir a todos los ciudadanos sin excluir a nadie.

Al respecto, tanto la Ley 23/1991 de acuerdo con Zapata (2015) como la Guía Institucional de Conciliación en Materia Civil (2007), marcan el inicio de una serie de importantes disposiciones legislativas que dan lugar a la utilización de determinados mecanismos alternativos de solución de controversias y, sobre todo, al establecimiento de la conciliación. Estas son algunas leyes colombianas que promueven el proceso de conciliación:

- Ley 270 de 1996, artículos 8 y 13.3.

- Ley 446 de 1998.

- Ley 446/1998, Parte III Mecanismos alternativos de resolución de conflictos.

- Título I de la Conciliación, artículos 64110.
- Ley 640/2001, Estatuto de Conciliación, artículos 2, 19, 27,35 y 38 .

- Ley 794 de 2003, artículo 44.

- Ley 906/2004, Código de Procedimiento Penal. Capítulo II Conciliación antes del Proceso, artículo 522. Capítulo III Mediación, artículos 523-527.

- Ley 975/2005, Ley de Justicia y Paz.

- Ley 1098/2006, Código la Infancia y la Adolescencia.

- Ley 1123/2007, Código Disciplinario del Abogado, literal 13, Artículo 28.

- Constitución Política, artículo 116. Inciso 4. En él se establece que los particulares podrán administrar justicia transitoriamente.

- Código de Procedimiento Civil, artículos 396 y 408 , entre otros.

En cuanto a la difusión de estas leyes y de los MASC el gobierno colombiano ha creado una división en el Ministerio de Justicia y la Ley, además cuenta con la Guía Institucional de Conciliación en Civil (2007) para informar a la población sobre mecanismos alternativos de resolución de conflictos en Colombia. Esta sección ministerial es también responsable de adoptar una posición jurídica sobre la interpretación y aplicación de las normas, principalmente en el ámbito de la conciliación extrajudicial, conciliadores y centros de conciliación. Esta división es conocida como el Programa Nacional de Conciliación.

Vale destacar que, en el marco de la conciliación, según la Ley Colombiana ésta se clasifica en judicial o extrajudicial en derecho o equidad se encuentra en derecho o en equidad. Así, relativo a la conciliación 
extrajudicial en equidad, ésta busca resolver conflictos atendiendo razones de justicia común y de igualdad entre las partes con la presencia de conciliadores en equidad, aquí se cuenta, además, con jueces de paz y conciliadores en derecho. Esta conciliación también recibe el nombre de justicia comunitaria, considerada como una característica conciliatoria más importante en Colombia.

Según el Ministerio de Justicia y Derecho (2014), los conciliadores en equidad se consideran un mecanismo alternativo de solución de conflictos por medio del cual dos o más personas resuelven sus controversias por intermedio de un tercero neutral llamado conciliador en equidad, persona que goza de reconocimiento comunitario social, que administra justicia de manera gratuita, motivando a los involucrados en un conflicto para que alcancen por si mismos o de mutuo acuerdo, una solución. Esta conciliación se considera un MASC auto compositivo como lo establece el artículo 116 inciso 4, de la Constitución Política Colombiana (1991), es decir; si las partes involucradas llegan por sí mismas a un acuerdo acerca de sus diferencias, sea, para que en la consecución de dicho acuerdo intervenga o no un tercero, quien, de existir, sólo se limita a la orientación del proceso más no a la resolución unilateral del mismo.

En este orden de ideas, Castro y Jaramillo (2018) explican que la justicia comunitaria en equidad es una modalidad de administración de justicia mediante la cual se faculta a operadores de la comunidad para que tramiten y decidan conflictos relevantes ante el orden jurídico del Estado. Así, la justicia comunitaria es una herramienta para implementar y fortalecer la conciliación en equidad, pensada para que cualquier miembro de la comunidad pueda acceder al sistema de justicia, acercando la justicia a los criterios populares de equidad al exigir que las decisiones sean tomadas por miembros de la propia comunidad, bajo tradiciones y prácticas propias. Por consiguiente, se le llama en equidad por cuanto, las decisiones que se toman cuentan, de manera predominante con la norma social pero enmarcada en un estatuto legal que las hace válidas ante el sistema judicial nacional (Ardila, 2016).

Con relación a la equidad, se resaltan algunas de las bases legales que le dan sustento, el artículo 85, modificado según el artículo 108: Principios de informalidad y celeridad para el logro de arreglos amistosos.

- Decreto 2350 de 2003 del Ministerio del Interior y de Justicia. Artículo 11 y siguientes: conciliación en los conflictos de los organismos de acción comunal.

- Ley 23 de 1991. Artículo 83: Gratuidad de la conciliación en equidad.

Finalmente, se advierte que también aplica la conciliación en equidad dentro de los municipios y zonas de influencia, se cuenta para ello con el Programa Nacional de Justicia y Equidad, que tiene la misión de fortalecer y consolidar los MASC para que cumplieran su objetivo que es desarrollar la convivencia comunitaria, utilizando entre otros el criterio de la equidad como una herramienta esencial de su aplicación, en especial, la figura de la conciliación en equidad. Bajo esta perspectiva, se planteó que para el año 2019 todos los municipios del país contarán con 
conciliadoras y conciliadores en equidad de manera organizada (Ministerio de Justicia y del Derecho)

\subsection{La conciliación como una estrategia de culfura de paz}

A pesar de décadas de violencia en Colombia, cesaron parcialmente las confrontaciones armadas, pero no todavía la cultura de la violencia que se volvió cotidiana. Después de estos períodos de paz relativa se inicia una nueva etapa de desafíos para poder prevenir nuevos conflictos en el país. Ahora bien, para el desarrollo del posconflicto armado, es necesario entenderlo en primera instancia como una combinación, entre el tiempo en que se inicia el alto al fuego de las hostilidades entre las partes previamente enfrentadas (Calderón, 2016). Es aquí donde los mecanismos de solución de conflictos entre ellos preponderantemente la conciliación influyen en conjunción con la administración de justicia dentro de los territorios vulnerables, al establecerse planes de acción que permitan lograr posibilidades de seguir avanzando en la pacificación integral del país.

Al referirnos al posconflicto revolucionario, debemos entender a la etapa siguiente como la reconstrucción y la búsqueda del estado natural de las cosas antes de sufrir las confrontaciones, reconstruir las poblaciones devastadas por la lucha armada, y su rehabilitación para poder avanzar a nuevas formas de dirección del Estado y participación ciudadana, proceso que será largo y costoso desde el punto de vista de la inversión económica y social (Calderón, 2016). Ciertamente, para lograr la reconstrucción y la rehabilitación, se necesitan de innovadoras estrategias que ayuden a consolidar una cultura de paz, en la que los mecanismos alternativos juegan un papel transcendental en la resolución de controversias de manera pacífica, buscando que las disputas se resuelvan de la mejor manera, otorgando mayores beneficios sin que las partes lleguen a la confrontación gracias a una participación decidida e intervención de jueces de paz, conciliadores en equidad y conciliadores en derecho.

El acto de conciliación busca potenciar la convivencia pacífica entre sus coasociados y la vigencia de un orden justo, convirtiéndose en instrumento eficaz para la disminución de la violencia pues permite tranquilizar los ánimos de las partes inmersas en los conflictos, y permite, además, que los ciudadanos obtengan respuestas en el corto plazo y todos los involucrados participen en la construcción de acuerdos. Se observa tambien que existen centros de conciliación públicos los cuales prestan un servicio gratuito y están integrados por un profesional en derecho para la mediación de acuerdos para el beneficio de todos.

La conciliación se considera un insumo para construir políticas públicas, el artículo 19 de la Ley 640 de 2001 señala los asuntos sobre los que se puede conciliar, esto es sobre todas las materias susceptibles de transacción, desistimiento y conciliación. Además de poderse conciliar asuntos civiles, comerciales, laborales, penales, administrativos, de convivencia, incluyendo la materia familiar. Se educa a los ciudadanos para la búsqueda de soluciones a los conflictos con el apoyo 
de varias autoridades facultadas para esta conciliación, según el artículo 31 de la Ley 640 de 2001.

\section{METODOLOGÍA}

Esta investigación es de carácter documental y se enmarca en el paradigma socio-crítico. Este se fundamenta en la crítica social con un marcado carácter autorreflexivo (Cebotarev, 2003). Igualmente, de acuerdo con Grijalba, Mendoza, y Beltrán (2020) el pensamiento sociocrítico consiste en: (...) "la examinación detallada y contextualizada de la situación o problemática de la realidad social a entender, que promueva la idea de vivir juntos, en favor de la realización de cambios necesarios para la superación de las problemáticas existentes" (p. 3).

De la misma manera, Arnal (1992) define el paradigma socio crítico como un proceso de reconstrucción de saberes la cual debe ser colectiva, donde se problematice a través del pensamiento dialéctico y crítico las realidades para su transformación. A su vez, tiene como objetivo, según Alvarado y García (2008), promover las transformaciones sociales, para dar respuesta a problemas específicos presentes en el seno de las comunidades, pero con la necesaria participación de sus miembros.

\section{REFLEXIONES FINALES}

En resumen, el propósito de los mecanismos de solución de conflictos implementados por Colombia no se limita a descongestionar al poder judicial de la saturación de casos. Para Colombia los MASC son vistos como una forma de participación e invitación a los ciudadanos colombianos para resolver las disputas que se susciten entre ellos y que se fomente la construcción de opciones pacíficas. En este sentido, es indiscutible el aspecto democrático y educativo de los MASC en la medida en que dan espacios de intervención a la comunidad en el desarrollo de la función judicial e impulsar la cultura de la paz.

De esta manera, es necesario, para lograr la reconstrucción y la rehabilitación de las comunidades afectadas por el conflicto armado, la puesta en marcha de estrategias puntuales que ayuden a consolidar la cultura de paz y que se promuevan los mecanismos alternativos para la resolución de conflictos de manera pacífica, sin llegar a la confrontación de las partes gracias a la apropiada intervención de jueces de paz, conciliadores en equidad y conciliadores en derecho.

No obstante, la promoción de la conciliación, a pesar de su reglamentación, no ha conseguido la disminución de las problemáticas sociales y humanas, puesto que, es necesario insistir en el cambio y evolución de las estructuras mentales, así como la renovación del pensamiento, ya que son herramientas básicas y esenciales para la armonía social, la comprensión y el sostenimiento equitativo de derechos y obligaciones en la comunidad. Estas acciones de pacificación son parte de los esfuerzos orientados a reafirmar la fe en la resolución pacífica y positiva de los conflictos con mira a la transformación benéfica para la sociedad en su conjunto, ello se podrá en la medida en que las políticas sociales sobre cultura de paz, y las políticas 
de educación para la paz hagan eco para la construcción de ciudadanos formados en el nuevo paradigma de hacer la paz y no la guerra.

Los esfuerzos de la institución colombiana para fortalecer la cultura de paz y los MASC a través de las políticas sociales se ha podido constatar en los gobiernos municipales los cuales han incorporado a sus funciones sociales, las plazas laborales de mediadores, conciliadores y árbitros, éstas son ahora mayores y existe, por tanto, la necesidad de preparación de expertos en esta área emergente del conocimiento y de participación y concientización ciudadana.

\section{TRABAJOS CITADOS}

Adams D. (1992). El Manifiesto de Sevilla sobre la Violencia: preparar el terreno para la construcción de la paz. Congreso Internacional sobre la paz en la mente de los Hombres: Declaración de Yamusukro; Declaración de Sevilla sobre la violencia. (1989) Recuperado en: https://unesdoc.unesco.org/ark:/48223/pf0000094314_ spa

Alvarado, L., \& García, M. (2008). Características más relevantes del paradigma socio-crítico: su aplicación en investigaciones de educación ambiental y de enseñanza de las ciencias realizadas en el Doctorado de Educación del Instituto Pedagógico de Caracas. Sapiens. Revista Universitaria de Investigación, 9 (2), pp. 187-202. Recuperado en: https://www.redalyc.org/ pdf/410/41011837011.pdf

Ardila Amaya, E. (2016). De la justicia judicial a la justicia comunitaria. Tesis doctoral. Departamento de Derecho Internacional, Eclesiástico y Filosofía del Derecho Getafe-Madrid. Recuperado en: https://core.ac.uk/download/pdf/79176651.pdf
Arnal, J. (1992). Investigación educativa. Fundamentos y metodología. Barcelona: Labor.

Audrerie, A. (2015) Médiation et conciliation: quelle distinction en matière juridique? Contribution à l'étude des modes alternatifs de règlement des conflits en droit social. Mémoire de recherche. Sous la direction de Mme Lise CASAUX-LABRUNEE Année universitaire 2014-2015. Université Toulouse 1 Capitole Master 2 Droit du Travail et de l'Emploi. Recuperado en: www.fenamef.asso.fr/ mediation-familiale/publications.../19

Barenboim, C. (2012). Políticas públicas urbanas e instrumentos de regulación en la Ciudad de Rosario. Revista Iberoamericana de Urbanismo, (7), pp. 31-41. Recuperado en: URI http://hdl.handle.net/2099/12539 ISSN20136242

Cabello Tijerina, P.A., Carmona Valdés, S.E., Gorjón Gómez, F. J., Iglesias Ortuño, E., Sáenz López, K.A. y Vázquez Gutiérrez, R.L. (2016). Cultura de paz. UANL. Colección Formación General Universitaria. Ciudad de México: Grupo Editorial Patria.

Cabello Tijerina, P.A., \& Quiñones-Díaz, K. A. (2019). La relevancia de la perspectiva territorial y femenina en la construcción de paz en Colombia. Convergencia Revista de Ciencias Sociales, 26 (80), pp. 1-25. Recuperado en: https://convergencia.uaemex.mx/article/ view/10286/9715

Calderón Rojas, J. (2016). Etapas del conflicto armado en Colombia: hacia el posconflicto. Latinoamérica. Revista de Estudios Latinoamericanos, (62), pp. 227257. Recuperado el 17 de febrero de 2021 en: http:// www.scielo.org.mx/scielo.php?script=sci_arttext\&pi$\mathrm{d}=$ S1665-742016000100227\&lng=es\&tlng=es.

Capella Riera, J. \& Tueros Way, E. (2005). Felipe MacGregor: Vida y legado de un maestro. Revista Educación, 14 (27), pp. 7-22. Recuperado el 19 de febrero en: http:// revistas.pucp.edu.pe/index.php/educacion/article/ view/8345/8652

Castro Herrera, F., \& Jaramillo Marín, J. (2018). La justicia en equidad y las violencias contra las mujeres en Colombia. 
Revista de derecho, 31 (2), pp. 115-133. Recuperado en: https://dx.doi.org/10.4067/S0718-09502018000200115

Cebotarev, E. A. (2003). El Enfoque Crítico: Una revisión de su historia, naturaleza y algunas aplicaciones. Revista Latinoamericana de Ciencias Sociales, Niñez y Juventud, 1(1), pp. 17-56. Recuperado el 06 de marzo de 2018 en: http://www.scielo.org.co/scielo.php?script=sci_arttextpid=S1692-715X2003000100002\&lng=en\&tlng=es.

Constitución Política de Colombia (1991).

Decreto 2350. (20 agosto de 2003) Diario Oficial No. 45.287, de 22 de agosto de 2003. Ministerio del Interior y de Justicia por el cual se reglamenta la Ley 743 de 2002.

Fisas Armengol, V. (2010). El proceso de paz en Colombia. Cuadernos de Construcción de la Paz (17). Barcelona: Escola de Cultura de Pau (ECP). Recuperado en: https:// escolapau.uab.cat/img/qcp/procesos_paz_colombia. pdf

Galtung, J. (1981). La violencia y sus causas. Contribución específica de la irenología al estudio de la violencia: tipologías. París. Francia: UNESCO.

--------(1995) Violencia, Paz e Investigación sobre la paz en Investigaciones teóricas. Sociedad y cultura contemporáneas. Madrid: Tecnos.

-(1998).Tras la violencia, 3R : reconstrucción, reconciliación, resolución : afrontando los efectos visibles e invisibles de la guerra y la violencia. Bilbao, España: Gernika Gogoratuz. Recuperado en: https://www.gernikagogoratuz.org/wp-content/uploads/2020/05/RG06completo.pdf

Giesecke, M. (1999). Cultura de paz y enseñanza de la historia. (pp. 303-316) en Bonilla A. Ecuador-Perú. Horizontes de la negociación y el conflicto. Quito, Ecuador: FLACSO. Recuperado el 15 de marzo del 2018 en: https://biblio.flacsoandes.edu.ec/libros/digital/50982.pdf

Guía Institucional de Conciliación en Civil (2007). Ministerio de Justicia y la Ley. Colombia: Universidad Nacional de Colombia. Recuperado en: https://www.udea. edu.co/wps/wcm/connect/udea/5fadc291-4ea0-4cfa-
b054-3cc111122b24/GuialnstitucionalDeConciliacionCivil_MinJusticia.pdf?MOD=AJPERES

Grijalba Bolaños, J., Mendoza Otero, J. y Beltrán Alonso, H. (2020). La formación del pensamiento sociocrítico y sus características: necesidad educativa en Colombia. Revista Universidad y Sociedad, 12(1), pp. 64-72. Recuperado el 17 de febrero de 2021, en: http://scielo.sld.cu/ $\mathrm{pdf} /$ rus/v12n1/2218-3620-rus-12-01-64.pdf

Harto de Vera, F. (2016). La construcción del concepto de paz: paz negativa, paz positiva y paz imperfecta. Cuadernos de estrategia. (183), pp. 119-146. Recuperado en: https://dialnet.unirioja.es/ejemplar/451439

Jiménez Bautista, F. (2014). Paz neutra: Una ilustración del concepto. Revista de Paz y Conflictos. (7), pp. 19-52. Recuperado en: https://revistaseug.ugr.es/index.php/revpaz/article/view/1806/2337

Johnson, K. (26 junio de 2017). Los disidentes de las FARC: ¿Cuántos son? ¿Cómo manejarlos? Razón pública. Recuperado en: https://razonpublica.com/los-disidentes-de-las-farc-cuantos-son-como-manejarlos/

Ley 23 (21 de marzo de 1991), Reglamentada por el Decreto Nacional 800 de 1991.Por medio de la cual se crean mecanismos para descongestionar los Despachos Judiciales, y se dictan otras disposiciones. Artículo 83: Gratuidad de la conciliación en equidad. Congreso de Colombia.

Ley 270 (07 de marzo de 1996). Estatutaria de la Administración de Justicia. Congreso de Colombia.

Ley 446 (07 de julio de 1998). Por la cual se adoptan como legislación permanente algunas normas del Decreto 2651 de 1991, se modifican algunas del Código de Procedimiento Civil, se derogan otras de la Ley 23 de 1991 y del Decreto 2279 de 1989, se modifican y expiden normas del Código Contencioso Administrativo y se dictan otras disposiciones sobre descongestión, eficiencia y acceso a la justicia. Concordancias: Decreto Reglamentario 1829 de 2013: Por el cual se reglamentan algunas disposiciones de las Leyes 23 de 1991, 446 de 1998, 640 de 2001 y 1563 de 2012. Congreso de Colombia. 
Ley 640 (05 Enero de 2001). Por la cual se modifican normas relativas a la conciliación y se dictan otras disposiciones. Congreso de Colombia.

Ley 794 (08 de enero de 2003). Derogado por el literal c), art. 626. Ley 1564 (12 de julio de 2012. Por la cual se modifica el Código de Procedimiento Civil, se regula el proceso ejecutivo y se dictan otras disposiciones. Congreso de Colombia.

Ley 906 (31 de agosto de 2004). Código de Procedimiento Penal. Capítulo II Conciliación antes del Proceso; Artículo 522. Capítulo III Mediación, artículos 523-527. Congreso de Colombia.

Ley 975 (25de julio de 2005). Ley de Justicia y Paz. Diario Oficial 45.980. Congreso de Colombia.

Ley 1098 (08 de noviembre de 2006). Código de la Infancia y la adolescencia. Diario Oficial No 46.446 Congreso de Colombia.

Ley 1123 (22 de enero de 2007). Código Disciplinario del Abogado, literal 13 Artículo 28. Diario Oficial. № 46.519 de 22 de enero de 2007. Congreso de Colombia.

Lopera Morales, J. J. (Mayo-Agosto 2011). Aproximación a la Justicia Transicional: interrogantes sobre su aplicabilidad en Colombia. Revista Diálogos de Derecho y Política. 2 (7), pp. 2145-2784. Recuperado en: https://revistas.udea.edu.co/index.php/derypol/article/ view/11064/10146

López Becerra, M. H. (julio - diciembre 2011). Teorías para la paz y perspectivas ambientales del desarrollo como diálogos de imperfectos. Revista Luna Azul. (33), pp. 85-96. Recuperado en: https://www.redalyc.org/ pdf/3217/321727235008.pdf

Maggiolo I. y Perozo Maggiolo J. (2007). Políticas Públicas: proceso de concertación Estado-Sociedad. Revista Venezolana de Gerencia 12 (39), pp. (373-392). Recuperado en: https://www.redalyc.org/pdf/290/29014474004. pdf

Martínez Guzmán, V. (septiembre-diciembre 2000). Saber hacer las paces. Epistemologías de los estudios para la paz. Convergencia, Revista de Ciencias Sociales. Revista de Ciencias Sociales, 7 (23), pp. 49-96. Recuperado en: https://www.redalyc.org/pdf/105/10502303.pdf

Ministerio de Justicia y del Derecho. (2008). Manual para la formación de conciliadores y conciliadores en equidad en Colombia. Bogotá: Corporación Razón Publica. Recuperado en: https://www.minjusticia.gov.co/Sala-de prensa/PublicacionesMinJusticia/MANUAL\%20 PARA\%20LA\%20FORMACI\%C3\%93N\%20DE\%20CONCILIADORES.pdf

Molina Cano, J. (julio-diciembre 2014). Gaston Bouthoul y el fenómeno-guerra. Revista Brasileira de Estudos Políticos. (109), pp. 197-224. Belo Horizonte, Brazil. Recuperado en: https://doi.org/10.9732/P.0034-7191.2014v109p197

Muñoz Muñoz, F. A. (1998). La paz imperfecta, apuntes para la reconstrucción del pensamiento pacifista. Revista Papeles de Cuestiones Internacionales (65), pp. 11-14. Recuperado en: https://www.fuhem.es/papeles_ articulo/la-paz-imperfecta-apuntes-para-la-reconstruccion-del-pensamiento-pacifista/

Muñoz Muñoz, F. A. y López Martínez, M. (2000) Historia de la paz. Tiempos, espacios y actores. Colección Monográfica "EIRENE" 12. Granada, España: Universidad de Granada.

Organización de las Naciones Unidas por la Educación, la Ciencia y la Cultura (UNESCO, 2019). Cultura de paz. Recuperado en: https://es.unesco.org/themes/programas-construir-paz

Real Academia Española. (2006). Diccionario de la lengua española. (22. ${ }^{a}$ edición). Madrid, España. Recuperado en: https://www.rae.es/obras-academicas/diccionarios/diccionario-esencial-2006

Rodríguez Jares. X (1999). Educación para la paz: su teoría y su práctica. 2 ed. Madrid: Popular. Recuperado en: https://www.redalyc.org/pdf/105/10503313.pdf

Rojas Dávila, R. (2014). La cultura de paz en el derecho internacional público: una breve introducción. Recuperado en: http://www.oas.org/es/sla/ddi/docs/publicaciones_digital_XLI_curso_derecho_internacional_2014_ Roberto_Rojas_Davila.pdf

Terminski, B. (enero - diciembre, 2010). The evolution of the concept of perpetual peace in the history of political-le- 
gal thought. Revista Perspectivas Internacionales., (1), pp. 277-290. Recuperado en: https://revistas.uniandes. edu.co/doi/pdf/10.7440/colombiaint77.2013.06

Ugarriza Uribe, J. E. (2013). La dimensión política del postconflicto: discusiones conceptuales y avances empíricos. Revista Colombia Internacional. (77) pp. 141-176. Recuperado en: https://revistas.uniandes.edu.co/doi/ pdf/10.7440/colombiaint77.2013.06

Valdivieso Collazos, A. M. (2012). La justicia transicional en Colombia. Revista Papel Político. 17 (2), pp. 621-653. Recuperado en: https://revistas.javeriana.edu.co/index. php/papelpol/article/view/6545

Zapata Gloria, E. (2012). La conciliation en Colombie. Mémoire présenté à la Faculté des études supérieures Université du Montréal en vue de l'obtention du grade de Maîtrise en droit, option droit international Recuperado en: https://papyrus.bib.umontreal.ca/xmlui/bitstream/ handle/1866/9616/Zapata_Gloria_Estella_2012_memoire.pdf?sequence $=2 \&$ isAllowed $=y$

\section{Gladis Ruiz Gómez}

Abogada de la Universidad Simón Bolívar, con Maestría en Derechos Humanos y Democracia de la Universidad Alcalá de Henares. Magister en Derecho Procesal de la Universidad de Medellín. Doctora en Filosofía con Acentuación en Ciencia Política de la Universidad Autónoma de Nuevo León de México. Miembro de la $\mathrm{JCl}$ y de la Red Justicia ATR, Profesora Universitaria, Investigadora y conferencista. Email: gladis.ruiz@unisimonbolivar.edu.co gladis_ruiz_gomez@hotmail.com. ORCID ID: https:// orcid.org/0000-0002-4295-6014

\section{Mireya Pérez Núñez}

Catedrática de la Universidad de la Costa, sede Sabanalarga. Dra en Sociología, (Universidad de Paris VII). Email: mireiopuntacardon@gmail.com/mperez93@cuc.edu.co 